\title{
Movement disorder: A manifestation of HIV and its response to therapy
}

\author{
Charulata S. Sankhla, Rajeev N. Soman ${ }^{1}$, Neha N. Gupta ${ }^{1}$, Pratik V. Shah ${ }^{1}$ \\ Departments of Neurology and ${ }^{1}$ Medicine, P.D. Hinduja Hospital, Mumbai, India
}

\begin{abstract}
Address for correspondence:
Dr. Sankhla Charulata,

Department of Neurology,

P.D. Hinduja National Hospital,

Veer Savarkar Marg,

Mahim - 400 016, Mumbai, India.

E-mail: charusankhla@gmail.com
\end{abstract}

DOI: $10.4103 / 0028-3886.59480$

\begin{abstract}
Both akinetic and hyperkinetic movement disorders may rarely be the presenting feature of human immunodeficiency virus (HIV) infection. The possible pathogenic basis is the involvement of subcortical structures by the HIV infection-related pathology. Opportunistic infections, or mass lesions complicating HIV infection. In addition dopaminergic dysfunction and medications may also play a role. We report a HIV infected male who presented with progressive choreoathetoid movements and dystonia. He had remarkable improvement of the movement disorder with tetrabenazine and anti-retroviral therapy (HAART) treatment.
\end{abstract}

Key words: Anti-retroviral therapy, human immunodeficiency virus, movement disorder

\section{Introduction}

Movement disorders, both akinetic and hyperkinetic, have been reported in 2-3\% of patients with human immunodeficiency virus (HIV) infection. ${ }^{[1]}$ The movement disorders include: Hemichorea, ballismus, myoclonus, tics, dystonia, tremors and Parkinsonism. ${ }^{[2]}$ In some of the reported cases, a specific pathology, such as toxoplasmosis involving the basal ganglia has been identified and in the cases where no such pathology was demonstrable, the movement disorder was attributed to HIV infection related pathology. Recognizing the movement disorder is important, because it may be the presenting manifestation of HIV infection. ${ }^{[3]}$

\section{Case Report}

A 47 year-old male, farmer by occupation, presented with progressive asymmetric involuntary movement involving the right upper limb followed by left upper limb, distal more than proximal, of 18 months duration. The movements were present at rest as well as during activity and were absent during sleep. The patient also had memory impairment and slurring of speech and slowness of activities. Two years before the present admission he had right sided weakness from which he recovered completely and eight years before he had two episodes of herpes zoster infection (by history), first on the left lower limb and the second on the trunk, within a short span of one month. The patient was a chronic alcoholic till six months before the present admission and used to consume $60 \mathrm{ml}$ of country liquor every day for twenty years. There was no history of receiving any antipsychotic or antiemetics medication and family history was unremarkable.

Physical examination revealed no signs of cutaneous or oral mucosal infection. He was conscious and co-operative patient and had a score of $21 / 30$ on mini-mental status examination (MMSE). He in addition had complex movement disorder: Asymmetric choreoathetoid movements of both upper limbs (left more than right) with a ballistic and dystonic component in the left upper limb. He also had head titubation and right sided pyramidal signs. His gait was some what slow and broad-based with tendency to fall to left side.

All the laboratory tests including thyroid profile and hematological works up were normal. Serology for HIV type 1 infection was positive, CD 4 count was 371 cell/cu $\mathrm{mm}$ and viral load was 3830 copies/ml. Toxoplasma antibody IgG titer was $300 \mathrm{IU} / \mathrm{L}$. He was also found to be positive for HBsAg however replication markers (HBeAg and HBV-DNA) were negative. Serum VDRL and TPHA were negative. 
Magnetic resonance imaging (MRI) of brain, T2 weighted and FLAIR images revealed cerebral atrophy with hyperintense lesion involving left cerebellar hemisphere, left brachium pontis, bilateral thalami, and left fronto-parietal lobes. No mass lesions were identified [Figures 1 and 2]. Magnetic resonance angiography (MRA) and venography (MRV) were unremarkable. Electro-encephalography (EEG) showed slow background activity with no interictal epileptic form discharges.

Patient was started on tetrabenazine and antiretro viral treatment (HAART). During the follow-up at four months he showed remarkable improvement in his involuntary movements, especially hemiballismus, gait and balance.

\section{Discussion}

The complex movement disorder in our patient was related to HIV infection and was the presenting feature. Hemiballismus, hemichorea and tremors are the most frequently reported hyperkinetic movement disorders. ${ }^{[2,4]}$ Most often the patient with movement disorders also have features of involvement of other components of neuroaxis.

The pathogenesis of movement disorder in HIV infection remains unclear. Opportunistic infections involving the subcortical structures are the common cause of movement disorders. ${ }^{[2]}$ HIV infection-related pathology involving basal ganglia and brainstem may also result in movement disorders. ${ }^{[2,4]}$ Autopsy studies showed histological evidence of subacute encephalitis in $90 \%$ of patients and in $77 \%$ of the patient there was involvement of basal ganglia, mainly putamen. ${ }^{[5,6]}$ A calcific vasculopathy has been described in the basal ganglia, predominantly involving the putamen and the outer segment of the globus pallidus. ${ }^{[2]}$ About $96 \%$ of patients with neuro-AIDS had showed evidence for

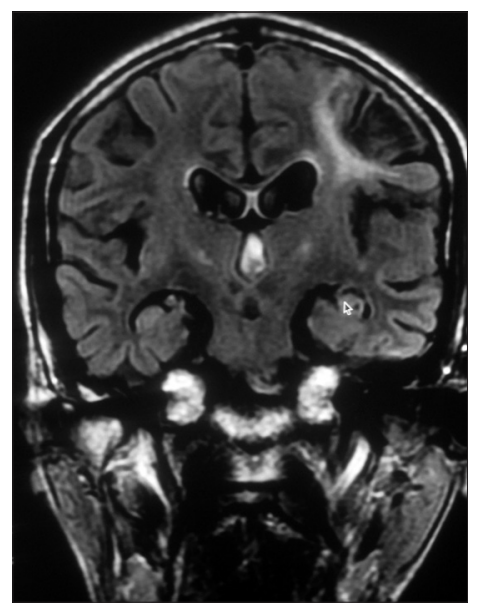

Figure 1: Coronal FLAIR magnetic resonance imaging scan showing hyperintense signal abnormalities in left frontoparietal region left cerebellum, left brachiopontis and bilateral thalamus. Note the diffuse cortical atrophy and ventricular enlargement central synthesis of HIV-specific IgG ${ }^{[2]}$ Ischemic infarction is common in patients with HIV infection and is related to hypercoaguable state associated with HIV infections. Hypercoaguable states in patients with HIV infection has been attributed to protein $C$ and protein $S$ deficiency, increases in factor VIII, presence of anticardiolipin antibodies and also to elevated homocystein levels. ${ }^{[7]}$

In HIV-1 infection, activated brain macrophages and microglia release quinolinic acid, a neurotoxin and N-methyl-D aspartate (NMDA) receptor agonist. Elevated cerebrospinal fluid quinolinic acid levels are associated with region specific cerebral volume loss in HIV infection and has been implicated in the development of cognitive deficits. ${ }^{[8]}$ Fluorodeoxyglucose positron emission tomography (FDG-PET) studies have shown relative hypermetabolism in the basal ganglia and the thalmi in patients with AIDS dementia complex with motor disorders. FDG-PET studies have also shown global cerebral hypometabolism in advanced HIV infection. ${ }^{[9]}$ Patients with HIV infection show increased sensitivity for extrapyramidal side-effects of neuroleptic drugs. ${ }^{[10]}$ Some of these changes may be contributing factors in the pathogenesis of movement disorders in HIV infection.

We have not done the cerebral functional studies, cerebrospinal fluid biochemical studies, and tests for prothrombotic state in our patient. MRI brain showed extensive subcortical lesions including both the thalami. The pathological basis for the MRI changes can be HIV infection-related pathology and also cerebral ischemia. He had history of focal deficits two years before the present admission. The anatomical substrate for the complex movement disorder in this patient may be related to the involvement of different projection fibers between the basal ganglia structures and the cortex.

Treatment of HIV-related extrapyramidal symptoms has uncertain efficacy. Chorea may respond to dopamine

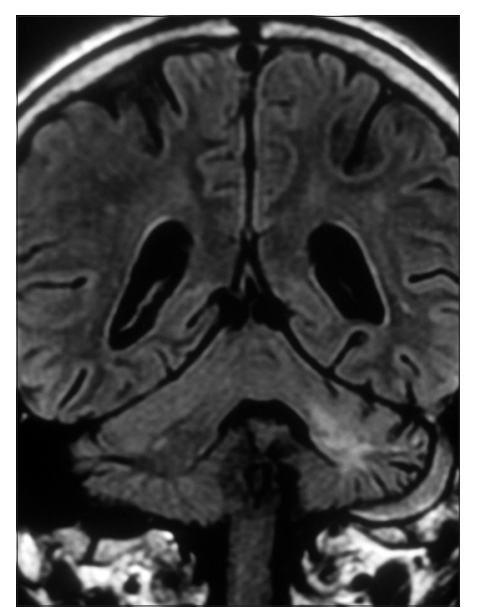

Figure 2: Coronal FLAIR magnetic resonance imaging scan showing hyperintense signal abnormalities in left cerebellum 
receptor blockade, but tremors, Parkinsonism and other movement disorders usually fail to respond to available therapies ${ }^{[5]}$ However in our patient there was significant improvement with the symptomatic therapy and HAART.

\section{References}

1. Cardoso F. HIV-related movement disorders; epideomology, pathogenesis and management. CNS drugs 2002;16:663-8.

2. Nath A, Jankovic J. Motor disorders in patient with HIV infections. Prog AIDS Pathol 1989;1:159-66.

3. Passarin MG, Alessandrini F, Nicolini GG, Musso A, Gambina G, Moretto G. Reversible choreoathetosis as the early onset of HIV encephalopathy. Neurol Sci 2005;26:55-6.

4. Gallo BV, Shulman LM, Weiner WJ, Petito CK, Berger JR. HIV Encephalitis presenting with severe generalised chorea. Neurology 1996;46:1163-5.

5. de la Monte SM, Ho DD, Schooley RT, Hirsch MS, Richardson EP Jr. Subacute encephalomyelitis of AIDS and its relation to HTLV-III infection. Neurology 1987;37:562-9.

6. Navia BA, Cho ES, Petito CK, Price RW. The AIDS dementia complex II. Neuropathology AnnNeurol 1986;19:525-35.

7. Fauci Anthony S, Lane H. Clifford. Human Immunodeficiency Virus: AIDS and related disorders. Harrison's principle of internal medicine; 17 Edition,volume 1;182:1180.

8. Heyes MP, Ellis RJ, Ryan L, Childers ME, Grant I, Wolfson T, et al. Elevated cerebrospinal fluid quinolinic acid levels are associated with region-specific cerebral volume loss in HIV infection. Brain 2001;124:1033-42.

9. Factor SA, Troche-Panetto M, Weaver SA. Dystonia in AIDS: Report of four cases. Mov Disord 2003;18:1492-8.

10. Hollander H, Golden J, Mendelson T, Cortland D. Extrapyramidal symptoms in AIDS patients given low dose metaclopramide or chlorpromazine. Lancet 1985;2:1186.

Accepted on 06-10-2009

Source of Support: Nil, Conflict of Interest: None declared. 